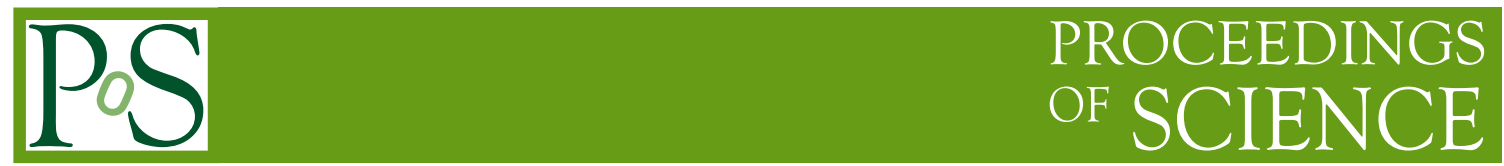

\title{
Observation of new charmonium (-like) decays
}

\section{Tong Liu, for the BESIII Collaboration*}

Institute of High Energy Physics, UCAS, Beijing 100049, China

E-mail: liutong2016aihep.ac.cn

In the past few years, a large number of unexpected new states, named as "charmonium-like states" or "XYZ states", have been found above the open charm threshold. These exotic states contains a charm anti-charm quark pair, but cannot be simply described as $c \bar{c}$ bound states. In this proceeding, we review recent results of $X Y Z$ studies by the BESIII experimen$\mathrm{t}$, including the following analyses: $X(3872) \rightarrow \omega J / \psi, X(3872) \rightarrow \pi^{0} \chi_{c 1}, Y(4220) \rightarrow \omega \chi_{c 0}$, $e^{+} e^{-} \rightarrow \pi^{+} \pi^{-} \psi(3770) \rightarrow \pi^{+} \pi^{-} D \bar{D}$ and $Z_{c}(3900) \rightarrow \rho \eta_{c}$

XXIX International Symposium on Lepton Photon Interactions at High Energies - LeptonPhoton2019 August 5-10, 2019

Toronto, Canada

${ }^{*}$ Speaker. 


\section{Intrduction}

In the quark model, a meson consists of one quark and one anti-quark, and a baryon is composed of three quarks. However, QCD also allows the existence of other exotic hadron states, like tetra-quark and penta-quark [1]. The first $X Y Z$ states, $X(3872)$, was found in the process $B^{ \pm} \rightarrow K^{ \pm} X(3872)$ with $X(3872) \rightarrow \pi^{+} \pi^{-} J / \psi$ by the Belle Collaboration in 2003 [2]. After that, a lot more states beyond the theoretical prediction and their properties have been found and studied in charmonium mass region, showing that they are good candidates of exotic hadrons.

The large data samples collected by the BESIII detector at the Beijing Electron Positron Collider (BEPCII) in the center-of-mass energy region between 3.6 and $4.6 \mathrm{GeV}$ provide a good opportunity to study such $X Y Z$ state. In this paper, we review recent results of $X Y Z$ study in BESIII.

\section{X states}

Previous study of $X(3872) \rightarrow \pi^{+} \pi^{-} J / \psi$ shows that the $\pi^{+} \pi^{-}$system is dominated by the $\rho$ resonance. Considering that no iso-spin partners is found in proton anti-proton collision, the process of $X(3872) \rightarrow \rho J / \psi$ violates iso-spin conservation. Meanwhile, $X(3872) \rightarrow \omega J / \psi$ conserves iso-spin and is supposed to have large branching ratio. The hadron molecule model predicts that measuring the decay rate precisely would help to determine the ratio of various components.

Using a $11.6 \mathrm{fb}^{-1}$ data sample collected with BESIII from 4.008 to $4.600 \mathrm{GeV}, X(3872)$ is observed for the first time in the $\omega J / \psi$ system with significance larger than $5 \sigma$ [3]. To completely describe the $\omega J / \psi$ mass spectrum, at least one additional Breit-Wigner resonance $X(3930)$ is needed. Depending on fit models, the resonant parameters of $X(3930)$ are $3932.6 \pm 8.7 \pm 4.7 \mathrm{MeV} / c^{2}$ and $59.7 \pm 15.5 \pm 3.7 \mathrm{MeV}$ (Fig. 1, top left), or $3926.4 \pm 2.2 \pm 1.2 \mathrm{MeV} / c^{2}$ and $3.8 \pm 7.5 \pm 2.6 \mathrm{MeV}$ (Fig. 1, top right). The resonant parameters of $X(3930)$ agree with $Y(3940)$ and $X(3915)$ observed by Belle and BarBar. The relative decay ratio $\mathscr{B}(X(3872) \rightarrow \omega J / \psi) / \mathscr{B}\left(X(3872) \rightarrow \pi^{+} \pi^{-} J / \psi\right)$ is measured to be $1.6_{-0.3}^{+0.4} \pm 0.2$, which agrees with BarBar's result $0.8 \pm 0.3$. The center-of-mass dependent cross section of $e^{+} e^{-} \rightarrow \gamma X(3872)$ is described using a single Breit-Wigner resonance $Y(4200)$ successfully. Using simultaneous fit to the cross section distributions of $X(3872) \rightarrow \omega J / \psi$ and $\pi^{+} \pi^{-} J / \psi$ (Fig. 1, bottom), the mass and width of the BW are determined to be $4200.6_{-13.3}^{+7.9}$ $\mathrm{MeV} / c^{2}$ and $115_{-26}^{+38} \mathrm{MeV}$, respectively, which agree with the resonant parameters of $Y(4260)$.

Using $9.0 \mathrm{pb}^{-1}$ data collected with a center-of-mass between 4.15 and $4.30 \mathrm{GeV}$, BESIII group search the processes $e^{+} e^{-} \rightarrow \gamma \pi^{0} \chi_{c J}$ and a new decay channel $X(3872) \rightarrow \pi^{0} \chi_{c 1}$ is observed for the first time, with the relative decay ratio $\mathscr{B}\left(X(3872) \rightarrow \pi^{0} \chi_{c 1}\right) / \mathscr{B}\left(X(3872) \rightarrow \pi^{+} \pi^{-} J / \psi\right)=$ $0.88_{-0.27}^{+0.33} \pm 0.10$ [4]. If assuming $X(3872)$ were $\chi_{c 1}(2 P)$, theory calculation in Ref. [5] predicts $\Gamma\left(X(3872) \rightarrow \pi^{0} \chi_{c 1}\right)$ as $0.06 \mathrm{keV}$, thus the total width of $X(3872)$ would be $1.0-2.0 \mathrm{keV}$, which is much narrower than other charmonium states.

\section{Y states}

Y states are produced directly in $e^{+} e^{-}$annihilation, so they have quantum number $J^{P C}=1^{--}$. The first $Y$ state is $Y(4260)$, observed by BarBar in the process $e^{+} e^{-} \rightarrow \gamma_{I S R} \pi^{+} \pi^{-} J / \psi$, then later $Y(4360)$ was found in $\pi^{+} \pi^{-} \psi(3686)$ spectrum. Unlike the charmonium states, these $Y$ states 

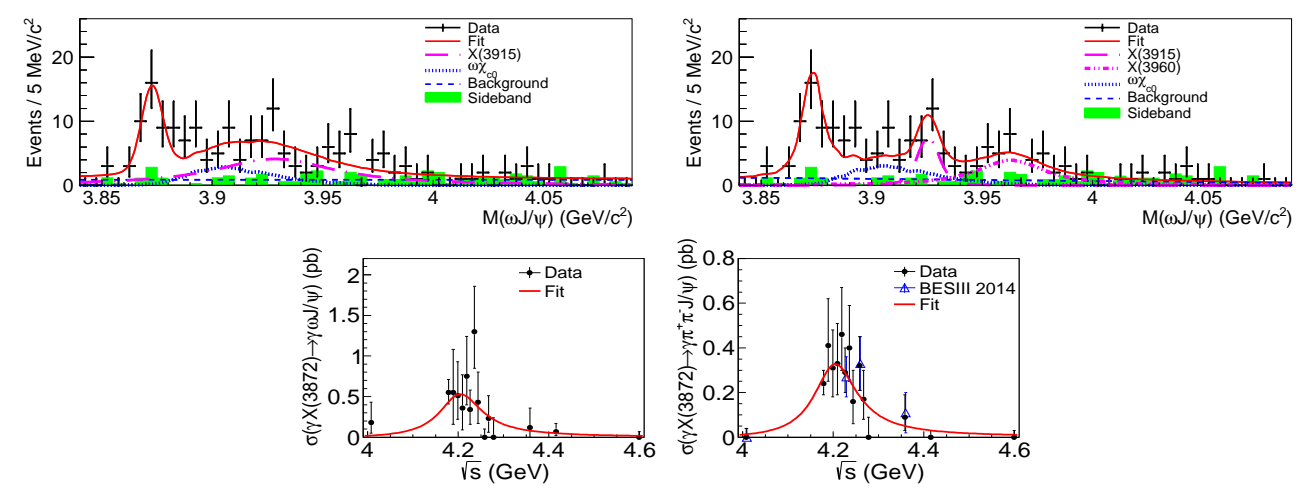

Figure 1: The $M_{\omega J / \psi}$ distribution and the fit using different fit model (top). The $\sqrt{s}$ dependent cross section of $e^{+} e^{-} \rightarrow \gamma X(3872) \rightarrow \gamma \omega J / \psi$ (bottom left) and $e^{+} e^{-} \rightarrow \gamma X(3872) \rightarrow \gamma \pi^{+} \pi^{-} J / \psi$ (bottom right).

strongly coupled to hidden-charm final states, so they are good candidates for exotic particles. Recently, according to a precise cross section measurement for $e^{+} e^{-} \rightarrow \pi^{+} \pi^{-} J / \psi$ reported by BESIII, two structures were observed. Resonant parameters of the first one are measured to be $4.220 \pm 3.1 \pm 1.4 \mathrm{MeV} / c^{2}$ and $44.1 \pm 4.3 \pm 2.0 \mathrm{MeV}$, respectively, at the same location as $Y(4260)$ but much narrower. The second resonance have mass and width as $4320.0 \pm 10.4 \pm 7.0 \mathrm{MeV} / c^{2}$ and $101.4_{-19.7}^{+25.3} \pm 10.2 \mathrm{MeV}$, agree with the $Y(4360)$ state. BESIII also measured the cross section of $e^{+} e^{-} \rightarrow \pi^{+} \pi^{-} h_{c}$ and open-charm final state $D^{0} D^{*-} \pi^{+}$, and both studies see two resonances in the distribution of $\sqrt{s}$ dependent cross section.

Using $7 \mathrm{fb}^{-1}$ data sample with a center-of-mass energy from 4.178 to $4.278 \mathrm{GeV}$, BESIII has recently reported a measurement of resonant parameters of $Y(4220)$ through $e^{+} e^{-} \rightarrow \omega \chi_{c 0}$ process [6]. The comparison of the measured resonant parameters of $Y(4220)$ in different channels is shown in Fig. 2 (left). It is hard to draw a conclusion that the structures are from the same resonance; the precision would need to be improved. Angular distribution measurement shows that the statistical significance of a combination of S- and D-wave contributions over a pure S-wave contribution is $2 \sigma$.
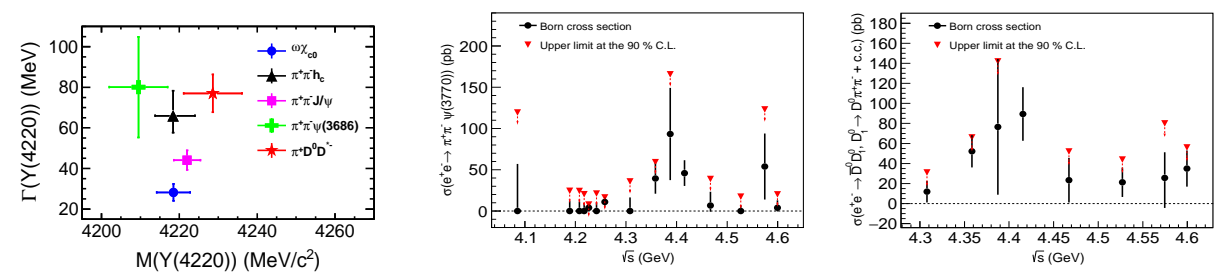

Figure 2: Left: Resonant parameters measured from different processes. Middle: Cross sections of the process $e^{+} e^{-} \rightarrow \pi^{+} \pi^{-} \psi(3770)$. Right: Cross sections of the process $e^{+} e^{-} \rightarrow D_{1}(2420)^{0} \bar{D}^{0}$.

Considering the observation of $Y$ states in $\pi^{+} \pi^{-} J / \psi$ and $\pi^{+} \pi^{-} \psi(3686)$ final states, a natural extension would be $\pi^{+} \pi^{-} \psi(3770)$. As described in Ref. [7], BESIII has studied the process $e^{+} e^{-} \rightarrow \pi^{+} \pi^{-} D \bar{D}$ using data sample from 4.09 to $4.60 \mathrm{GeV}$, the $\pi^{+} \pi^{-} \psi(3770)$ signal has been observed for the first time, no significant signal of $\pi^{+} \pi^{-} \psi\left(1^{3} D_{3}\right)$. The cross section of 
$e^{+} e^{-} \rightarrow \pi^{+} \pi^{-} \psi(3770)$ is measured and a structure is seen at $4.4 \mathrm{GeV}$ (Fig. 2, middle). Based on the current statistics, it's hard to conclude the structure is from $Y(4360)$ or $\psi(4415)$. In $\pi^{ \pm} \psi(3770)$ mass spectrum, we see hints of $Z_{c}$ states at 4.04 and $4.13 \mathrm{GeV}$. Reference [8] suggests that $Y(4260)$ is a relative $\mathrm{S}$-wave system of $D_{1}(2420) \bar{D}+D \bar{D}_{1}(2420)$. In the analysis of $e^{+} e^{-} \rightarrow \pi^{+} \pi^{-} D \bar{D}$, a $7.4 \sigma D_{1}(2420)$ signal is observed for the first time in $\pi^{+} \pi^{-} D$ mass spectrum, but no fast rise is seen above $D_{1}(2420) \bar{D}$ threshold (Fig. 2 (right)). In $D \bar{D}$ mass spectrum, we have looked at the heavy quark-spin-symmetry partner of $X(3872)$, named $X_{2}(4013)\left(J^{P C}=2^{++}\right)$, whose existence would be a strong support for $X(3872)$ being a molecule. However, the results are negative.

\section{Z states}

$Z_{c}(3900)^{ \pm}$state was firstly observed by BESIII and then confirmed by Belle in $\pi^{ \pm} J / \psi$ spectrum through process $e^{+} e^{-} \rightarrow\left(\gamma_{I S R}\right) \pi^{+} \pi^{-} J / \psi$. Since it is charged and decays to $\pi^{ \pm} J / \psi$, there are at least four quarks $(c \bar{c}+u \bar{d}$ or $d \bar{u})$ in $Z_{c}(3900)$. Through a partial wave analysis of this process, the quantum number $J^{P}$ of $Z_{c}(3900)^{ \pm}$was determined to be $1^{+}$[9]. BESIII had also observed a neutral state $Z_{c}(3900)^{0}$ in the process $\pi^{0} \pi^{0} J / \psi$, suggesting $Z_{c}(3900)^{ \pm, 0}$ as iso-spin triplet. Considering that $Z_{c}(3900)$ sits slightly above the $D \bar{D}^{*}$ threshold, BESIII studied the process $e^{+} e^{-} \rightarrow \pi^{ \pm, 0}\left(D \bar{D}^{*}\right)^{\mp, 0}$ and a state with similar resonant parameters was observed in $\left(D \bar{D}^{*}\right)^{\mp, 0}$ mass spectrum. In the spectrum of $\pi^{ \pm} h_{c}$ through the process of $e^{+} e^{-} \rightarrow \pi^{+} \pi^{-} h_{c}$, BESIII observed another $Z_{c}$ state named $Z_{c}(4020)$, sitting $5 \mathrm{MeV}$ above $D^{*} \bar{D}^{*}$ threshold. Later this state was found in $\pi^{0} h_{c}$ and $\left(D^{*} \bar{D}^{*}\right)^{\mp, 0}$ decay modes.

According to the calculation described in [10], the relative decay ratio of $Z_{c}^{(\prime)} \rightarrow \rho \eta_{c}$ to $\pi J / \psi\left(\pi h_{c}\right)$ is sensitive to distinguish the tetra-quark model from molecule model of $Z_{c}$. Recently, an analysis of $e^{+} e^{-} \rightarrow \pi^{+} \pi^{-} \pi^{0} \eta_{c}$ was performed in BESIII. The decay $Z_{c}(3900) \rightarrow \rho \eta_{c}$ is seen with statistical significance $4.3 \sigma$, and there is no significant signal of $Z_{c}(4020)$. We also measure the relative decay ratio $\mathscr{B}\left(Z_{c}^{ \pm\left({ }^{\prime}\right)} \rightarrow \rho^{ \pm} \eta_{c}\right) / \mathscr{B}\left(Z_{c}^{ \pm\left(^{\prime}\right)} \rightarrow \pi^{ \pm} J / \psi\right)$. Comparison between this measurement and theoretical calculation is listed in Tab. 1. The measured $\mathscr{R}_{Z_{c}(3900)}$ is closer to the tetra-quark model, but the molecule model cannot be completely excluded. Reductions in the uncertainty of the theoretical calculation are needed.

Table 1: Comparison of the measured $\mathscr{R}_{Z_{c}(3900)}$ and $\mathscr{R}_{Z_{c}(4020)}$ with the theoretical predictions from Ref. [10]

\begin{tabular}{c|ccc|ccc}
\hline \hline$\sqrt{s} /$ model & $4.226 \mathrm{GeV}$ & $4.258 \mathrm{GeV}$ & $4.358 \mathrm{GeV}$ & Type-I & Type-II & Molecule \\
\hline $\mathscr{R}_{Z_{c}(3900)}$ & $2.2 \pm 0.9$ & $<5.6$ & $\ldots$ & $230_{-140}^{+330}$ & $0.27_{-0.17}^{+0.40}$ & $0.046_{-0.017}^{+0.025}$ \\
$\mathscr{R}_{Z_{c}(4020)}$ & $<1.6$ & $<0.9$ & $<1.4$ & $6.6_{-5.8}^{+56.8}$ & $0.010_{-0.004}^{+0.006}$ \\
\hline \hline
\end{tabular}

\section{Summary}

Here we review the recent results of $X Y Z$ states at BESIII experiment. The strange behavior of exotic particles have been studied and compared with theoretical prediction. However, there are still questions that are not clear. BESIII is planning to take data above $4 \mathrm{GeV}, 500 \mathrm{pb}^{-1}$ per point with a $10 \mathrm{MeV}$ interval in the next 5-10 years, while the maximum of $\sqrt{s}$ of BEPCII will be increased from 4.6 to $4.9 \mathrm{GeV}$ [1]. More exciting results from BESIII collaboration will come up soon. 


\section{References}

[1] Nora Brambilla, Simon Eidelman, Christoph Hanhart, Alexey Nefediev, Cheng-Ping Shen, Christopher E. Thomas, Antonio Vairo, Chang-Zheng Yuan, arXiv:1907.07583v1

[2] S.K.Choi et al. (Belle Collaboration), PRL 91, 262001 (2003).

[3] M. Ablikim et al. [BESIII Collaboration], PRL 122, 232002 (2019).

[4] M. Ablikim et al. [BESIII Collaboration], PRL 122, 202001 (2019).

[5] S. Dubynskiy and M.B. Voloshin, PRD 77, 014013 (2008).

[6] M. Ablikim et al. [BESIII Collaboration], PRD 99, 091103 (2019).

[7] M. Ablikim et al. [BESIII Collaboration], PRD 100, 032005 (2019).

[8] Q. Wang, C. Hanhart and Q. Zhao, PRL 111, 132003 (2013).

[9] M. Ablikim et al. [BESIII Collaboration], PRL 119, 072001 (2017).

[10] A. Esposito, A. L. Guerrieri and A. Pilloni, PLB 746, 194 (2015).

[11] M. Ablikim et al. [BESIII Collaboration], arXiv:1906.00831. 